\title{
EVIDENCIA FAUNISTICA DE INTENSIFICACION DE LAS CORRIENTES DE FONDO DE LA CUENCA DE PANAMA DURANTE UN ESTADIO GLACIAL.
}

por:

\section{Adolfo Molina Cruz*}

\author{
Ciencias Marinas Vol. 2 Núm. I
}

* Oregon Stote University. School of Oceanography, Corvallis Oregon, 97331, U.S.A. Becario de CONACYT.

\begin{abstract}
RESUMEN
Muchos sedimentos biogénicos silíceos en la cuenca de Panamá son removidos selectivamente ("winnowing") desde las cordilleras circundantes por corrientes de fondo. Tales corricntes parecen haber sido más intensas durante el último estadío glacial (Pleistoceno) que en el Holoceno. El núcleo analizado para este trabajo (Y69-7/P) muestra $0.015 \%$ (promediol de material retrabajado (radiolarios) en el Holoceno y $0.043 \%$ en la cima del Pleistoceno. Los porcentajes son significativamente diferentes $195 \%$ de confianza). El material retrabajado proviene del Terciario.
\end{abstract}

\section{ABSTRACT}

The distribution of much of the siliceous beiogenetic sediments is due to winnowing in the Panama Basin. This winnowing is produced by bottom currents. Such currents seem to be more intense during the last glacial luppermost Pleistocenel than in the Holocene interglacial. The analyzed core (Y $897 / P)$ in this paper shows $0.015 \%$ (averagel or reworked material (radiolarians) in the Holocene and $0.043 \%$ in the uppermost Pleistocene. The percentages are significantly different $195 \%$ of confidence). The reworked material comes from the Tertiary.

\section{INTRODUCCION}

Los geólogos marinos de Oregon State University han estado realizando estudios sobre algunos aspectos de'; sedimentación del fonclo marino en la cuenca de Panamá.

Se ha observado que la distribución de los sedimentos biogénicos es controlada por las corrientes de fondo; las que remueven selectivamente ("winnowing") las partículas desde las cordilleras circundantes (Van Andel, 1973, Moore et al., 19731.

El presente trabajo trata con microfósiles retrabajados (radiolarios) y esta- blece evidencia para sugerir que las corrientes de fondo han sido más intensas durante el último estadío glacial (Pleistoceno Superior) que en el Holoceno interglacial en la cuenca de Panamá.

Los radiolarios de los sedimentos cuaternarios de un núcleo (Y69-7IP) de la parte oeste de la cuenca de Panamá (lat. $0^{\circ} 6^{\prime} \mathrm{N}$ y long. $86^{\circ} 29^{\prime} \mathrm{W}$ ) han sido contados y el número de retrabajados referido.

La abundancia de especies del Terciario encontradas en el Holoceno $y$ en el Pleisioceno Superior son analizadas estadísticamente. 


\section{ANTECEDENTES:}

La cuenca de Panamá esta circundada al norte por Centro América, al este - por Sur América, y al oeste y sur por las cordilleras sumergidas "Cocos" y "Carnegie" respectivamente (Fig. 1). El entronque de estas dos cordilleras submarinas es marcado por las islas Galápagos. Al centro de la cuenca están situadas las cordilleras "Coiba" y "Malpelo" las que dividen físicamente a ésta en una parte profunda "Este" y una parte menos profunda "Oeste".

En la parte oeste de la cuenca de Panamá la mayoría de los sedimentos son de origen biógeno; sin embargo, su distribución no refleja el patrón de la fuen-. te de aporte (producción primaria) (Moore et al., 1973). También se nota que los. restos terrígenos gruesos (mayor que 149 micras) son raramente lo bastante abundantes como para enmascarar los efectos de disolución y retrabajo sobre las partículas biogénicas gruesas (mayor que 149 micras) (Kowsman, 1972).

La distribución del número de radiolarios en la porción gruesa ("coarse fraction") de los sedimentos (Fig. 2) muestra su más alto valor en el sur de la parte oeste de la cuenca y los valores más bajos sobre las cimas de las cordillera Cocos y Carnegie (Kowsman, 1972).

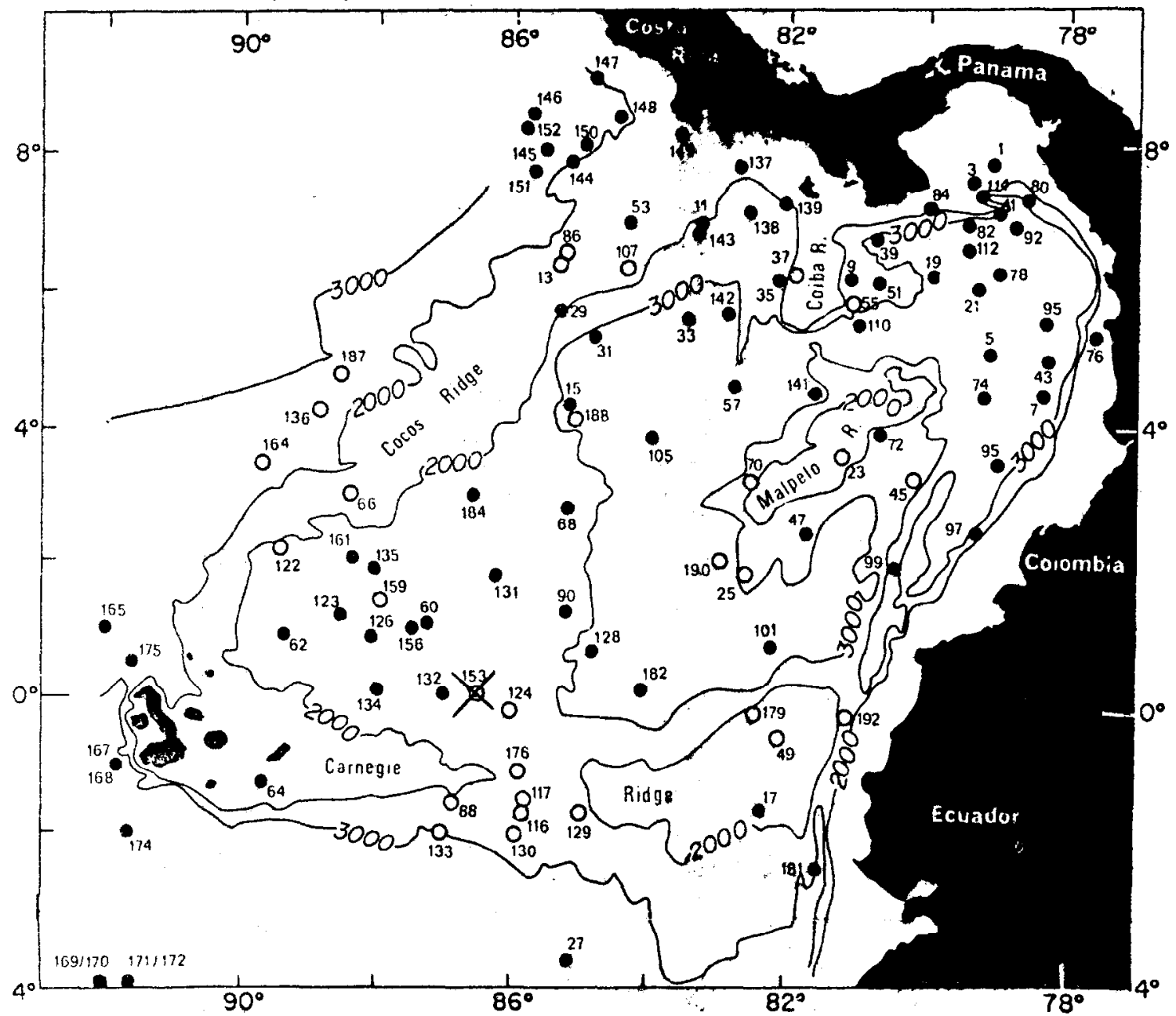

Figura 1. Localización de las muestras superficie. Los circulos abiertos representan muestras que contienen microfósiles retrabajados Contornos en metros (Reproducidos de Kowsmann, 1972) 


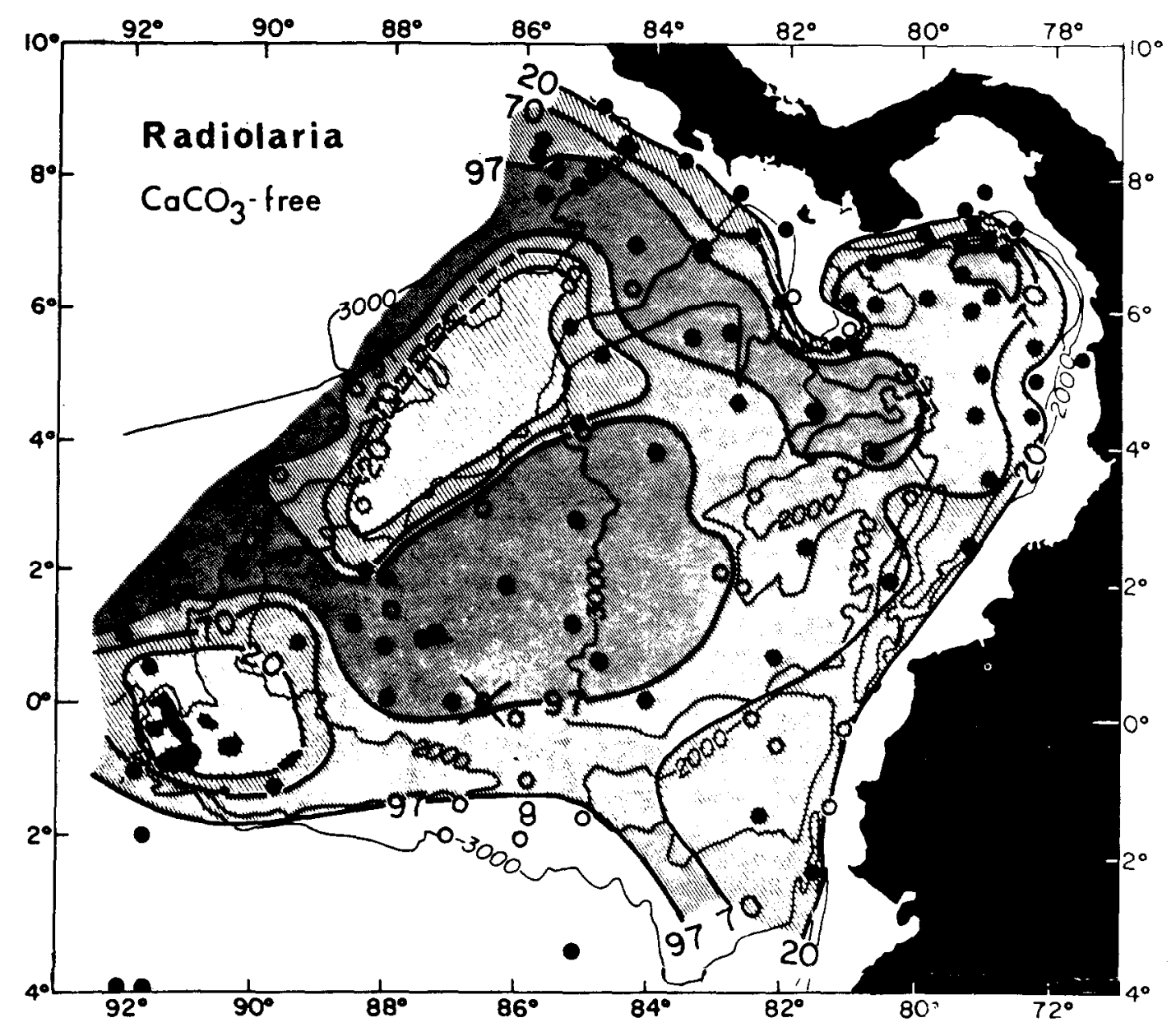

Figura 2. Distribución de los sedimentos superficíales de radiolarios (muestra burda libre de carbonatos). Los circulos abyertos como en la Figura 1. (Reproducida de Kowsmann. 1972)

$$
\text { \&Localización del núcleo Y69 - 71P }
$$

Las muestras de profundidades mayores que 2400 metros contienen los más altos promedios de la concentración de ópalo. Tales profundidades corresponden a los flancos de las cordilleras donde los radiolarios son depositados (Moore ef al., 1973).

Londslale y colaboradores (1972) han observado fuertes corrientes de fondo (> $15 \mathrm{~cm} / \mathrm{seg}$ ) producida por las mareas en la cuenca de Panamá $y$ han concluido que aunque tales corrrientes no son direccionales ni continuas, son to suficientemente fuertes para perturbar los sedimentos pelágicos y remover las partículas finas de áreas de alto relieve donde el flujo es canalizado. Ellos reportaron la presencia de "ripple marks" y dunas de arena foramináceas a lo largo de la silla central de la cordillera Carnegie (cerca del lugar donde el núcleo Y69-71P fue colectado).

Por consiguienté las corrientes de mareas $y$ el removimiento selectivo de partículas que ellas producen ("winnowing") son factores geológicos importantes para determinar la distribución regional de los sedimentos pelágicos. Si estos factores han variado a través del tiempo geológico un patrón sedimentario diferente puede estar presente en cada una de las edades geológicas. 


\section{METODOS Y RESULTADOS:}

Para determinar si las corrientes de fondo han variado en intensidad entre el último estadío glacial (Pleistoceno Superior) y el Holoceno interglacial; el núcleo de pistón Y69-71P fue seleccionado para análisis. Este núcleo fue muestreado en la latitud $0^{\circ} 6^{\prime} \mathrm{N}$ y longitud $86^{\circ} 29^{\prime} \mathrm{W}$ por un crucero oceanográfico de "Oregon State University" sobre el flanco norte de la cordillera submarina Carnegie (cerca a su "silla" central) en 1969 (Fig. 1).

El contacto Pleistoceno-Holoceno en el núcleo es asumido aproximadamente a $80 \mathrm{~cm}$ bajo la "tapa" del núcleo y la base del último estadío glacial aproximadamente a 4 metros (Dienkelman, 1973).

Seguidamente, dos series de muestras seleccionadas estadísticamente al azar fueron tomadas del núcleo. Una serie de cinco muestras para el intervalo 10-80 cm bajo la "tapa" del núcleo y otra de seis muestras para el intervalo 80-400 $\mathrm{cm}$. Las tablas para muestrear estadísticamente al azar de "Rand Corporation"
(1955) fueron usadas para determinar los niveles muestreados. La distribución de las muestras tomadas a lo largo del intervalo considerado del núcleo, es mostrada en la tabla No. 1.

Las muestras fueron lavadas con $\mathrm{HCl}$ y $\mathrm{H}_{2} \mathrm{O}_{2}$ para cemover el carbonato de calcio. Después, 44 placas con radiolarios (4 por cada nivel) fueron preparadas utilizando el método de Moore (1971). Este método asume que los especímenes sobre la placa presentan una distribución estadísticamente al azar.

Los radiolarios en cada placa fueron identificados y contados. El porcentaje de material retrabajado fue determinado por la cantidad de individuos extintos hace 400,000 años 0 antes (Stylatractus universus o más viejosl.

Los promedios de material retrabajado de las dos series de muestras fueron calculados. Estos son: $0.015 \%$ para el estadío interglacial (Holoceno) y $0.043 \%$ para el estadío glacial (Pleistoceno Superior).

Aunque tales medias estadísticas son muy pequeñas, la diferencia entre ellas

\begin{tabular}{|c|c|c|c|c|c|c|c|c|c|c|c|}
\hline \multirow{2}{*}{$\begin{array}{l}\text { Nivel } \\
\text { (cm) }\end{array}$} & \multicolumn{5}{|c|}{ No. de Radiolerioe Contado } & \multicolumn{5}{|c|}{ No. de Radiolarios Retrabajado } & \multirow{2}{*}{$\begin{array}{c}\% \\
\text { Retroboj } \\
\end{array}$} \\
\hline & Place & $\begin{array}{c}\text { Place } \\
2\end{array}$ & \begin{tabular}{|c|} 
Placa \\
3 \\
\end{tabular} & \begin{tabular}{|c|} 
Placa \\
4 \\
\end{tabular} & Total & $\begin{array}{c}1 \text { eca } \\
1\end{array}$ & $\begin{array}{c}\text { Placa } \\
2\end{array}$ & $\begin{array}{c}\text { Place } \\
3\end{array}$ & $\begin{array}{c}\text { Placa } \\
4\end{array}$ & Total & \\
\hline $18-20$ & 2510 & 2062 & 2529 & 2244 & 9545 & 1 & 1 & 0 & 0 & 2 & .0214 \\
\hline $26-28$ & 2206 & 1527 & 1447 & 1927 & $7^{107}$ & 0 & $o$ & o & 0 & 0 & $\mathbf{o}$ \\
\hline $42-44$ & 2052 & 1700 & 2327 & 2212 & 8219 & 1 & 0 & 0 & 0 & 1 & .0120 \\
\hline 66.68 & 1559 & 1860 & 1767 & 1786 & 6972 & 1 & - & 0 & 0 & I & .0286 \\
\hline $74 \cdot 76$ & 2.62 & 1527 & 1854 & 2007 & 7450 & i & 0 & o & 0 & 0 & .0134 \\
\hline $102-104$ & 2381 & 1642 & 1905 & 1693 & 7621 & 1 & 0 & 1 & 1 & 3 & .0393 \\
\hline $130-132$ & 1940 & 2119 & 1607 & 16,6 & 7282 & 1 & $\mathbf{1}$ & 1 & 1 & 4 & .0549 \\
\hline $158-160$ & 1962 & 1796 & 1844 & 1303 & 6895 & 1 & 1 & 0 & 1 & 3 & .0435 \\
\hline $220-222$ & 1005 & 1022 & 1174 & 926 & 4127 & 1 & 0 & 0 & 0 & I & .0242 \\
\hline $256-268$ & 1146 & 1364 & 1383 & 1233 & 5126 & 0 & 0 & 1 & 1 & 2 & .0390 \\
\hline $330-332$ & 2014 & 2192 & $\lg 6 g$ & 2228 & 8403 & 1 & 1 & 2 & 1 & 5 & .0595 \\
\hline
\end{tabular}


es significativa $195 \%$ de confianza) en acuerdo al análisis de variancia lprueba F) referido en Remington y Shork (1970).

$\mathrm{De}$ lo anterior se deduce que la cantidad de material trabajado es más abundante en el estadío glacial que en el interglacial.

En acuerdo con Nigrini (1971) los radiolarios encontrados corresponden a las biozonas: Buccionosphaera invaginata len lo más alto del cuaternario), Collosphaera tuberosa y Amphirhopalum ysillon. Nigrini estableció estas zonas de radiolarios con sedimentos cuaternarios del Océano Pacífico Ecuatorial.

Las especies de radiolarios más abundantes presentes en este estudio son:

\section{Tetrapyle octacantha \\ Ommatartus tefrathalamus \\ Lithelius minor \\ Theoconus minithorax \\ Spongopyle osculosa \\ Lithostrobus seriatus \\ Anthocyrtidium zanguebaricum \\ Pylospira octopyle \\ Larcopyle butschlii \\ Stylochlamydium asteriseus \\ Hexacontium encanthum}

El material retrabajado consiste de dos especies:

\section{Stichocorys delmontense $y$ Stichocorys peregrina.} perior.

Ambos provienen del Terciario Su-

\section{CONCLUSIONES:}

Las corrientes de fondo han estado presentes en la cuenca de Panamá durante el último estadío glacial (Pleistoceno Superior) y en el Holoceno (estadío interglaciall; ya que material retrabajado ha sido encontrado en ambos estadíos.

Puesto que la cantidad de dicho material es más abundante en el estadío glacial, se concluye que las corrientes de fondo que producen el retrabajo fueron más intensas en dicho estadío.

En grupos de radiolarios que difieren mucho de edad, los efectos de disolución pueden enmascarar el verdadero promedio de material retrabajado y conducir a conclusiones incorrectas (Moore, 1969). Sin embargo, ya que los cambios morfológicos de los radiolarios predominantes en el Pleistoceno Superior y en el Holoceno en la cuenca de Panamá han sido imperceptibles; y las especies retrabajadas han sido las mismas en ambos esradíos, se asume que los efectos de solución selectiva no han sido tan decisivos.

Yamashiro (1974) en su trabajo con foraminíferos ha sugerido que la redepositación de sedimentos controla más los patranes de distribución de éstos en la cuenca de Panamá que los efectos de disolución.

Johnson (1972) presentó una extensa teoría para explicar cambios en la circulación oceánica debido a glaciaciones. El propuso que la erosión substancial del fondo océánico (Pacífico Ecuatorial) ocurrió durante estadíos glaciares a causa de la intensificación de las corrientes de fondo.

Heath (1969) ha sugerido que las corrientes de fondo y la actividad bentónica pueden ser incrementadas cuando el volumen de agua profunda, siendo formada bajo la capa de hielo en la Antártica, crece durañte un estadío glacial. El ha señalado que los sedimentos del Terciario Superior y del Cuaternario del Pacífico Ecuatorial no están bien seleccionados; apoyando su teoría.

Pisias (1974) ha mostrado incremento de la proporción de ópalo en los sedimentos de la cuenca de Panamá durante estadíos glaciales y ha sugerido que esto es a causa del incremento de producción primaria debido a la intensificación de la circulación.

$\mathrm{Si}$ las corrientes de fondo han sido más intensas durante el último estadío glacial en la cuenca de Panamá, es posible que los sedimentos removidos (incluyendo los radiolarios del Terciario retrabajados) hayan sido transportados sobre distancias mós grandes. 


\section{AGRADECIMIENTOS}

Doy las gracias al Dr. Theodore C. Moore por toda la ayuda que me ha brindado leyendo este trabajo y haciéndome sugerencias. También, agradezco a la Escuela de Oceanografía de "Oregon State University" que me proporcionó las muestras para este estudio.

Hago extenso mi agradecimiento al Consejo Nacional de Ciencia y Tecnologla de México que. me sostiene económicamente mediante una beca.

El presente trabajo fue presentado en el $V$ Congreso Nacional de Oceanografía efectuado en Guaymas, Son., en octubre de 1974.

\section{BIBLIOGRAFIA}

Dinkelman, M., 1973. Lote Quaternary radiolarian, PaleoOceanography of the Panama Basin, Eastern Equa. torial Pacific: Ph. D. dissertation, Oregon State. Universify.

Heath, G. R., 1969. Carbonate sedimentotian in the abyssal equatorial Pacific during the past 50 million years: Geol. Soc. Am. Bull.: 80: 689-694.

Johnson, A. D., 1972. Qceon-Floor Erosion in the Equa- torial Pacific: Geol. Soc. Am. Bull: 83(10): 3121-3144. Kowsmann, R. D., 1972. Panamo Basin surface sedimens: coarse components: M. S. dissertation, Oregon State University.

Lonsdale. P., B. T. Malfait and F. N. Spiess, 1972: Abyssal sand waves on the Carnegie Ridge: Geol. Soc. Am. Abstracts $4(7)$ : 579-580.

Moore, T. C., Jr., G. R. Heath and R. D. Kowsmann, 1973. Biogenic sedimentation in the Panama Basin: J. of Geol. 81|4|: 458.

Moore, T. C., Jr., 1973. Method of randomly distributing grains for microscopic examination: J. of Sedimentary Petrol., 43(3): 904.

1969. Radiolaria: change in skeletal weight and resistance to solution: Geol. Soc. Am. Bull.: 80(10): 2103-2107.

Nigrini. C. A., 1971. Radiolarion zones in the Quaternary of the Equatorial Pacific Qcean. In: The Micropaleontology of the Oceans, Funnel, B. M. and Riedel, W. R., leds.) Combridge.

Pisias, N., 1974. Model of late Pleistocene-Holocene variations in rate of sediment accumulation: Panama Basin, Eastern Equatorial Pacific: M. S. dissertation. Oregon State University.

Remington, R. D. and M. A. Shork, 1970. Statistics with applications to the biological and health sciences. Prentice-Hall, Inc. Englewood Cliffs, New Jersey.

Rand. Corporation, 1955. Tables of Random Numbers; The Free Press of Glencoe, New York.

Van. Andel, Ti. H., 1973. Texture and dispersal of sediments in the Panama Basin (in press).

Yamashiro, C., 1974. Dirferentiating dissolution and transport effects in foraminiferal sedjments from the Panama Basin: J. Foraminiferal Spec., vol., in press. 